\title{
Accuracy and Optimization of a Subcutaneous Insulin Model for Less Acute Critical Care Patients*
}

\author{
Felicity Thomas, Jennifer Dickson, Chris Pretty, Kent Stewart, Liam Fisk, Geoffrey Shaw and J. \\ Geoffrey Chase, Member, IEEE
}

\begin{abstract}
Extending safe, effective glycemic control to the general wards requires a simple approach using subcutaneous (SC) insulin. However, this approach can increase relative risk compared to intravenous insulin due to the increased variability of SC insulin appearance. This paper evaluates the accuracy of a SC plasma insulin model and optimizes its parameters using measured plasma insulin data from 6 less acute critical care patients treated with SC insulin. The SC plasma insulin model used captures the dynamics of regular SC insulin well. However, there appears to be a positive bias leading to an overall median [IQR] residual error of -28.3 [-37 19] $\mathrm{mU} / \mathrm{L}$. The optimized model reduced the RMS residual error by $\mathbf{2 0 - 7 0 \%}$ for each patient. The distinct inter- and intrapatient, and cohort variation seen in this data highlights the importance to of understanding how SC insulin appearance dynamics may be affected by the subject condition.
\end{abstract}

\section{INTRODUCTION}

Stress-induced hyperglycaemia is prevalent in critical care, and can occur in patients with no history of diabetes [1-4]. Hyperglycaemia worsens outcomes, leading to further risk of complications, including sepsis [5], myocardial infarction [1], polyneuropathy, and multiple organ failure [2]. Adaptive model-based protocols for accurate glycaemic control (AGC) that modulate insulin and nutrition have shown considerable promise in the intensive care unit (ICU) [6-10]. However, they are not suitable for less acute wards because of the demands they place on clinical staff [11-14] and the lack of intravenous (IV) lines for IV delivery.

To extend safe, effective glycaemic control to the general wards, a simple approach using subcutaneous (SC) insulin delivery is necessary. However, there is an increased rick when using SC insulin as its appearance is much more variable than IV insulin appearance [15]. This increased variability compounds the existing difficulty of managing intra- and inter- patient variability.

In model-based control, plasma insulin dynamics have a significant impact of identified model parameters, such as

* Research supported by University of Canterbury doctoral scholarships

F. Thomas is with the Department of Mechanical Engineering, University of Canterbury, Christchurch, New Zealand (corresponding author phone: +64 $27 \quad 338 \quad$ 4087; e-mail: felicity.thomas@pg.canterbury.ac.nz

J. Dickson, C. Pretty, K. Stewart, L. Fisk, J. G. Chase , is with the Department of Mechanical Engineering, University of Canterbury, Christchurch, New Zealand (e-mail: Jennifer.dickson@pg.canterbury.ac.nz, kent.stewart@pg.canterbury.ac.nz, @ chris.pretty@canterbury.ac.nz, liam.fisk@pg.canterbury.ac.nz, Geoff.chase@ canterbury.ac.nz ).

G. Shaw, is with the Department of Intensive Care, Christchurch School of Medicine and Health Sciences, New Zealand (e-mail: Geoff.shaw@cdhb.health.nz ). insulin sensitivity (SI). Hence, reducing error in modelling plasma insulin appearance will reduce SI variability and error, and thus increase the accuracy and utility of the parameter for glycaemic control.

This paper evaluates the accuracy of a SC plasma insulin model and optimises model parameters for less acute critical care patients. Plasma insulin blood samples from an ongoing study of SC insulin delivery, in the Christchurch Hospital Intensive Care Unit (ICU) were used to evaluate this model.

\section{PATIENTS AND METHODS}

\section{A. Subcutaneous Insulin Study Patients}

A total of 6 patients enrolled in a prospective clinical trial studying a protocol for SC insulin delivery. Each patient had a set of blood samples assayed for insulin and C-peptide. Patients were included in the study if they were:

- Age $\geq 18$ years

- Expected to survive ICU care

- Expected ICU length of stay $\geq 72 \mathrm{hrs}$

- Receiving treatment by the tablet-based STAR AGC protocol (Stochastic TARgeted) (2 sequential BG measurements $\geq 8 \mathrm{mmol} / \mathrm{L}$ ) [16]

In a stable condition, likely to be discharged to less acute wards $<48 \mathrm{hrs}$

TABLE I. SUMMARY OF SUBCUTANEOUS TRIAL PATIENT CHARACTERISTICS. DATA ARE SHOW AS MEDIAN [IQR] WHERE APPROPRIATE.

\begin{tabular}{|l|c|}
\hline Number (N) & 6 \\
\hline Age (years) & $58[43-60]$ \\
\hline Gender (M/F) & $5 / 1$ \\
\hline APACHE II score & $18.5[14.3-23.5]$ \\
\hline Hospital mortality (L/D) & $(6 / 0)$ \\
\hline
\end{tabular}

Patients received 3 doses of SC insulin over 12 hours. Blood glucose (BG) was measured every 30 mins with a blood gas analyser (BGA, Radiometer ABL90Flex, Copenhagen). Insulin and nutrition doses were determined every 4 hours by an SC insulin protocol. This study was approved by the Upper South Regional Ethics Committee, New Zealand.

During each 12 hour trial, 18 blood samples were taken to assess plasma insulin concentrations. Samples were taken at times $\mathrm{t}=0,30,60,90,180,240$ mins where $\mathrm{t}=0$ mins marks the first SC insulin bolus given. Insulin concentrations were determined using immunometric assays (Elecsys 2010, Roche Diagnostics, Germany). The reported coefficient of variation $\left(\mathrm{CV}_{\mathrm{A}}\right)$ for the insulin $3.8 \%[17,18]$. 


\section{B. Model}

For this study, the clinically validated Intensive Control Insulin-Nutrition-Glucose (ICING) model of the glucoseinsulin system was used [19]. For forward simulation using regular SC insulin (e.g. Actrapid, Novo Nordisk, Denmark), an additional kinetics sub-model was added, modelling transport from the SC layer to the plasma[20].

$$
\begin{array}{cr}
\dot{x}_{d m}=U_{d m}+p_{d}\left(\frac{1}{a} x_{h}-Q \frac{x_{d m}^{3}}{V^{2}}\right)-k_{d m} x_{d m} & \text { Equation (1) } \\
\dot{x}_{h}=U_{h}-p_{d}\left(\frac{1}{\mathrm{a}} x_{h}-Q \frac{x_{d m}^{3}}{V^{2}}\right) & \text { Equation (2) } \\
\dot{x}_{\mathrm{i}}=k_{d m} x_{d m}-k_{\mathrm{i}} x_{\mathrm{i}} & \text { Equation (3) } \\
U_{R I}=f_{R I} k_{\mathrm{i}} x_{\mathrm{i}} & \text { Equation (4) }
\end{array}
$$

Where $x_{h}, x_{i}$ and $x_{d m}$ are the total insulin mass in the hexameric $(h)$, local interstitial, $(i)$, and dimeric or monomeric, $(d m)$, compartments, respectively [mU]. Transport parameters are denoted $k_{i}$ and $k_{d m}\left[\min ^{-1}\right]$, and $U_{d m}$ and $U_{h}$ are the exogenous appearance rate $\left[\mathrm{mUmin}^{-1}\right]$ as delivered by injection to the dimeric or monomeric and the hexameric compartment respectively. $p_{d}$ is the hexameric dissociation rate $\left[\mathrm{min}^{-1}\right]$. Exogenous insulin input is $U_{R I}$ $\left[\mathrm{mU} \cdot \mathrm{min}^{-1}\right]$, where the unique scaling factor, $f_{R}$, highlights the many currently unquantified processes at work during the absorption process. The parameters in Table II were identified using published clinical data to describe a typical plasma insulin profile for regular insulin [21]. The overall model describes the appearance a common of SC insulin type e.g. Actrapid (Novo Nordisk, Denmark).

TABLE II. FITTED VALUES OF SUBCUTANEOUS INSULIN MODEL CONSTANTS

\begin{tabular}{|c|c|}
\hline Parameter & Value \\
\hline $\boldsymbol{k}_{\tilde{i}}$ & $4.60 \times 10^{-2} \mathrm{~min}^{-1}$ \\
\hline $\boldsymbol{k}_{d m}$ & $1.20 \times 10^{-2} \mathrm{~min}^{-1}$ \\
\hline $\mathbf{Q}$ & $5.02 \times 10^{-10}$ \\
\hline $\boldsymbol{p}_{d}$ & 1 \\
\hline $\boldsymbol{f}_{R I}$ & 0.597 \\
\hline
\end{tabular}

\section{Analysis Methods}

Measured and modelled plasma insulin values were compared and residuals calculated:

$$
\text { Residual Error }=I(t)_{\text {measured }}-I(t)_{\text {modelled }} \quad \text { Equation (5) }
$$

where $I(t))_{\text {measured }}$ is the plasma insulin measured during the 12 hour trials and $\mathrm{I}(\mathrm{t})_{\text {modelled }}$ is the forward prediction of the plasma insulin using the model identified SI profile for these patients during the 12 hour trials. Residuals were first calculated using the model parameters outlined above. The root mean square (RMS) and the median and interquartile range (IQR) of the residual errors was calculated for each patient and the overall cohort. Finally, an optimal value of the parameter, $f_{R I}$ was identified by iteratively searching the range of 0.60 to 0.20 in steps of 0.01 to find the value of $f_{R I}$ that generated the smallest residuals and the best model fit.

\section{RESULTS AND DISCUSSION}

\section{A. SC Plasma Insulin Model}

The SC plasma insulin model as published by Fisk [20] with $f_{R I}=0.597$ captures the dynamics of the regular SC insulin used in this study well, as seen in Figure I. This result indicates the underlying model structure for regular insulin is correct. However, there appears to be a positive bias leading to an overall median [IQR] residual error of 28.3 [-37 -19] mU/L. All 6 patients demonstrated this bias with the modelled insulin being consistently higher than the measured insulin as seen in Table III.

The updated parameter of $f_{R I}=0.25$ significantly reduced the residual error with an overall RMS of 17.4 and median [IQR] of -5.37 [-15 9.7] $\mathrm{mU} / \mathrm{L}$. This value also eliminated model bias with 3 patients having a positive median residual and 3 patients have a negative median residual, resulting in a better overall model fit. RMS residual error was reduced by between $20 \%$ and $70 \%$ for each patient with this adjustment.

The original model value for $f_{R I}$ was found using data from 18 heathy male volunteers [20]. This change in $f_{R I}$ thus reflects a distinct difference in SC insulin kinetics between healthy volunteers and critical care patients. This parameter is equivalent to a unique clearance in the local interstitium. It lumps together difficult to quantify dynamics, such as clearance from the subcutaneous depot, affinity to antibodies (appearing as different volumes of distribution), and altered clearance routes. Therefore, the reduction of $f_{R I}$ suggests less of the injected SC insulin is appearing in critical care patients than would appear in a healthy individuals injected with the same SC insulin bolus.

This increased degradation of insulin in the local interstitium is likely to be due to the increased edema typical of critical care patients. The extra fluid at the injection site would slow the transport of insulin to the plasma, as well as allowing more insulin to be cleared via other pathways. In addition, 
the lack of movement and low skin temperatures found in the critically ill have been shown to slow the transport of insulin to the plasma $[15,22]$. The air temperature in the Christchurch ICU is a constant 16 degrees Celsius, and most patients are immobilized and only wearing hospital gowns, both of which are typical in this setting.

Patient condition also affects the glucose-insulin metabolism resulting in inter- and intra- patient variability as condition evolves [23]. This impact is evident in Figure I, where there are a wide range of plasma insulin response curves. For example, Patient 6 shows an acute spike in plasma insulin after the $3^{\text {rd }}$ bolus while Patient 5 shows slow insulin plasma clearance resulting in plasma insulin concentration building up over time.

TABLE III. RESIDUAL ERROR OF SC MEASURED - MODELLED PLASMA INSULIN. DATA PRESENTED AS MEDIAN[IQR].

\begin{tabular}{|c|c|c|c|c|}
\hline \multirow{2}{*}{$\begin{array}{c}\text { Residual } \\
\text { Error }\end{array}$} & \multicolumn{2}{|c|}{$f_{\boldsymbol{R I}}=\mathbf{0 . 5 9 7}$} & \multicolumn{2}{c|}{$\boldsymbol{f}_{\boldsymbol{R} I}=\mathbf{0 . 2 5}$} \\
\cline { 2 - 5 } & $\boldsymbol{R M S}$ & Median[IQR] & $\boldsymbol{R M S}$ & Median[IQR] \\
\hline $\begin{array}{c}\text { Patient } \\
\mathbf{1}\end{array}$ & 31.3 & $-31.6[-36-26]$ & 8.90 & $0.144[-4.97 .8]$ \\
\hline $\begin{array}{c}\text { Patient } \\
\mathbf{2}\end{array}$ & 36.2 & $-36.5[-44-29]$ & 21.7 & $-24.2[-28-12]$ \\
\hline $\begin{array}{c}\text { Patient } \\
\mathbf{3}\end{array}$ & 26.6 & $-17.8[-35-11]$ & 13.0 & $10.6[3.915]$ \\
\hline $\begin{array}{c}\text { Patient } \\
\mathbf{4}\end{array}$ & 24.9 & $-23.6[-27-19]$ & 12.1 & $4.26[-1210]$ \\
\hline $\begin{array}{c}\text { Patient } \\
\mathbf{5}\end{array}$ & 31.1 & $-22.8[-33-9.9]$ & 24.8 & $-7.58[-1425]$ \\
\hline $\begin{array}{c}\text { Patient } \\
\mathbf{6}\end{array}$ & 34.9 & $-30.8[-38-22]$ & 18.5 & $-13.0[-23-8.0]$ \\
\hline Overall & 31.1 & $-28.3[-37-19]$ & 17.4 & $-5.37[-159.7]$ \\
\hline
\end{tabular}

Hence to extend safe, effective glycemic control to the general wards, a simple approach using subcutaneous (SC) insulin delivery and a simple, generalizable SC insulin kinetic transport model are necessary. Such a model must also have the ability to account a range of patient conditions. The ability to separately account for the variability of SC insulin kinetics would enable similar, proven models and methods used for AGC in critical care with IV insulin delivery to be used for SC insulin delivery, with the addition of an SC insulin model that meets these criteria.

To achieve this latter outcome, $f_{R I}$ needs to be optimized over large range of patient data instead of having patient specific insulin clearance parameters. Variability can also be managed through conservative insulin dosing and robust protocols. However, the distinct inter-cohort variation seen in this data highlights the importance to of understanding how the SC dynamics may be affected by the cohort, and accounting for them directly. In addition, SC insulin models based on healthy, T2DM or critical care data should only be cautiously applied to different cohorts.
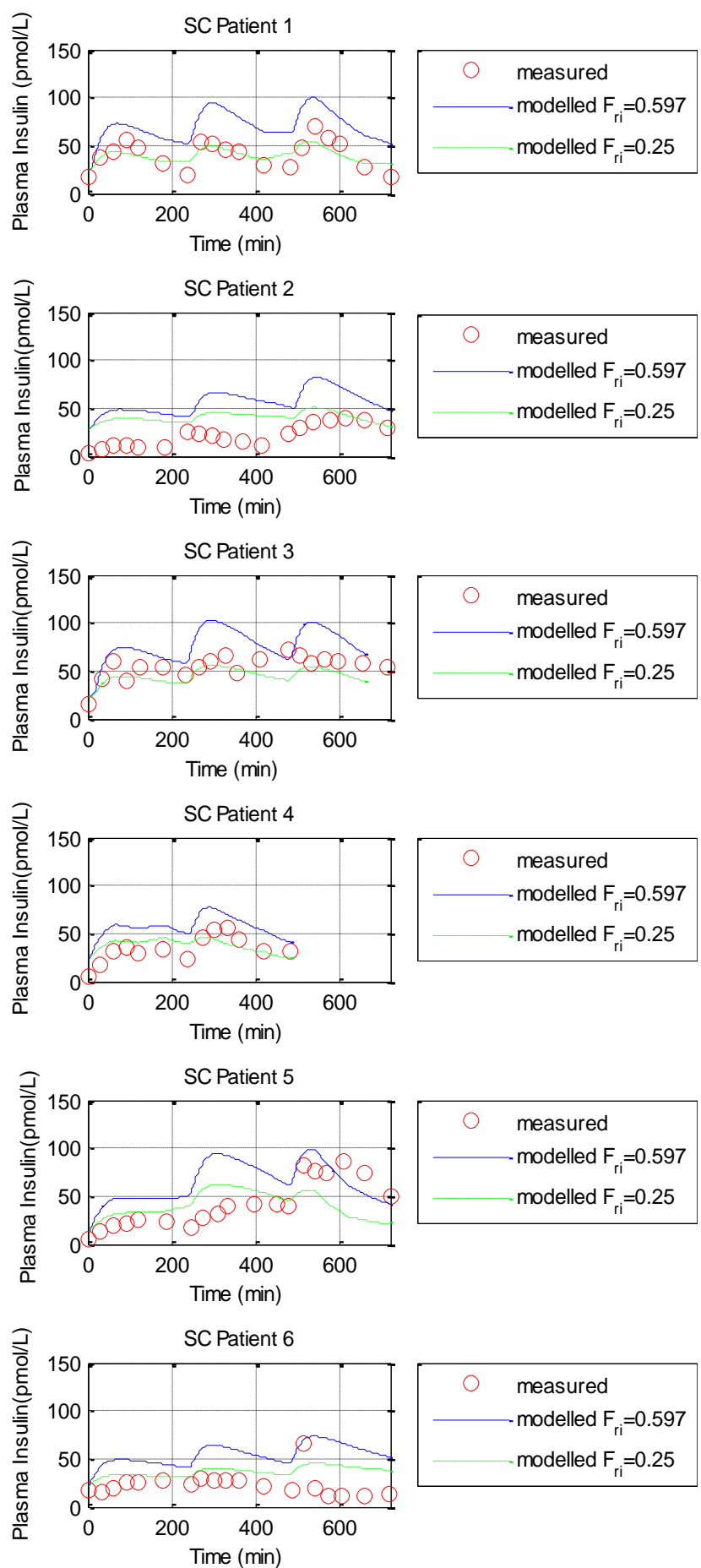

SC Patient 6

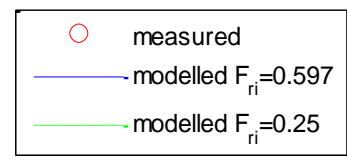

FIGURE I. MEASURED AND MODELLED PLASMA INSULIN DATA FOR SC INSULIN USING $F_{\mathrm{RI}}=0.597 \mathrm{AND} \mathrm{F}_{\mathrm{RI}}=0.25$ 


\section{CONCLUSION}

To extend safe, effective glycemic control to the general wards, a simple approach using subcutaneous (SC) insulin delivery is necessary. However, these changes increase the risk due to variability of the patient and the variability that can occur in SC insulin appearance [15]. The SC plasma insulin model evaluated captures the dynamics of the regular SC insulin well. However, there appears to be a positive bias leading to an overall median [IQR] residual error of -28.3 [37 -19] $\mathrm{mU} / \mathrm{L}$. The optimized model reduced the RMS residual error by between $20 \%$ and $70 \%$ for each patient. The distinct inter- and intra- patient and cohort variation seen in this data highlights the importance of understanding how SC dynamics may be affected by the subject condition, and accounting for this variability directly in the SC model to better generalize existing proven metabolic models used in critical care environments.

\section{ACKNOWLEDGMENT}

\section{Christchurch Hospital ICU Clinical team}

\section{REFERENCES}

[1] S. E. Capes, D. Hunt, K. Malmberg, and H. C. Gerstein, "Stress hyperglycaemia and increased risk of death after myocardial infarction in patients with and without diabetes: a systematic overview," Lancet, vol. 355, pp. 773-778, Mar 42000.

[2] G. van den Berghe, P. Wouters, F. Weekers, C. Verwaest, F. Bruyninckx, M. Schetz, et al., "Intensive insulin therapy in critically ill patients," N Engl J Med, vol. 345, pp. 1359-67, Nov 82001.

[3] B. A. Mizock, "Alterations in fuel metabolism in critical illness: hyperglycaemia," Best Pract Res Clin Endocrinol Metab, vol. 15, pp. 533-51, Dec 2001.

[4] K. C. McCowen, A. Malhotra, and B. R. Bistrian, "Stressinduced hyperglycemia," Crit Care Clin, vol. 17, pp. 107-124, Jan 2001.

[5] B. R. Bistrian, "Hyperglycemia and infection: which is the chicken and which is the egg?," JPEN J Parenter Enteral Nutr, vol. 25, pp. 180-181, Jul-Aug 2001.

[6] J. Lin, D. Lee, J. G. Chase, G. M. Shaw, A. Le Compte, T. Lotz, et al., "Stochastic modelling of insulin sensitivity and adaptive glycemic control for critical care," Computer Methods and Programs in Biomedicine, vol. 89, pp. 141-152, Feb 2008

[7] J. G. Chase, G. M. Shaw, J. Lin, C. V. Doran, C. Hann, T. Lotz, et al., "Targeted glycemic reduction in critical care using closedloop control," Diabetes Technol Ther, vol. 7, pp. 274-82, Apr 2005.

[8] A. Evans, A. Le Compte, C. S. Tan, L. Ward, J. Steel, C. G. Pretty, et al., "Stochastic targeted (STAR) glycemic control: design, safety, and performance," J Diabetes Sci Technol, vol. 6, pp. 102-15, Jan 2012.

[9] R. Hovorka, J. Kremen, J. Blaha, M. Matias, K. Anderlova, L. Bosanska, et al., "Blood glucose control by a model predictive control algorithm with variable sampling rate versus a routine glucose management protocol in cardiac surgery patients: a randomized controlled trial," J Clin Endocrinol Metab, vol. 92, pp. 2960-4, Aug 2007.

[10] R. Kulnik, J. Plank, C. Pachler, M. E. Wilinska, A. GroseljStrele, D. Rothlein, et al., "Evaluation of implementation of a fully automated algorithm (enhanced model predictive control) in an interacting infusion pump system for establishment of tight glycemic control in medical intensive care unit patients," $J$ Diabetes Sci Technol, vol. 2, pp. 963-70, Nov 2008.
[11] D. Aragon, "Evaluation of nursing work effort and perceptions about blood glucose testing in tight glycemic control," Am J Crit Care, vol. 15, pp. 370-7, Jul 2006.

[12] P. Carayon and A. P. Gurses, "A human factors engineering conceptual framework of nursing workload and patient safety in intensive care units," Intensive Crit Care Nurs, vol. 21, pp. 284 301, Oct 2005.

[13] J. Gartemann, E. Caffrey, N. Hadker, S. Crean, G. M. Creed, and C. Rausch, "Nurse workload in implementing a tight glycaemic control protocol in a UK hospital: a pilot time-in-motion study," Nurs Crit Care, vol. 17, pp. 279-84, Nov-Dec 2012.

[14] M. A. Malesker, P. A. Foral, A. C. McPhillips, K. J. Christensen, J. A. Chang, and D. E. Hilleman, "An efficiency evaluation of protocols for tight glycemic control in intensive care units," $\mathrm{Am}$ J Crit Care, vol. 16, pp. 589-98, Nov 2007.

[15] M. Berger, H. J. Cuppers, H. Hegner, V. Jorgens, and P. Berchtold, "Absorption Kinetics and Biologic Effects of Subcutaneously Injected Insulin Preparations," Diabetes Care, vol. 5, pp. 77-91, 1982.

[16] A. Evans, A. Le Compte, C. S. Tan, L. Ward, J. Steel, C. G. Pretty, et al., "Stochastic Targeted (STAR) Glycemic Control: Design, Safety, and Performance," Journal of Diabetes Science and Technology, vol. 6, pp. 102-115, 2012.

[17] Roche, "Data Sheet - C-Peptide Immunoassay, Elecsys 1010/2010/Modular Analytics E170," Roche Diagnostics, Mannheim, Germany 03184897 190, 2005.

[18] Roche, "Data Sheet - Insulin Immunoassay, Elecsys 1010/2010/Modular Analytics E170," Roche Diagnostics, Mannheim, Germany 12017547 122, 2004.

[19] J. Lin, N. N. Razak, C. G. Pretty, A. Le Compte, P. Docherty, J. D. Parente, et al., "A physiological Intensive Control InsulinNutrition-Glucose (ICING) model validated in critically ill patients," Comput Methods Programs Biomed, vol. 102, pp. 192205, May 2011.

[20] L. Fisk, "Model-Based Decision Support in Glycaemic Control," Doctor of Philosophy, Mechanical Engineering, University of Canterbury University of Canterbury 2014.

[21] Food and Drug Administration, "Insulin aspart clinical pharmacology and biopharmaceutics review(s) " Center for Drug Evaluation and Research1999.

[22] M. Berger, P. A. Halban, L. Girardier, J. Seydoux, R. E. Offord, and A. E. Renold, "Absorption Kinetics of Subcutaneously Injected Insulin - Evidence for Degradation at the Injection Site," Diabetologia, vol. 17, pp. 97-99, 1979.

[23] C. G. Pretty, A. J. Le Compte, J. G. Chase, G. M. Shaw, J. C. Preiser, S. Penning, et al., "Variability of insulin sensitivity during the first 4 days of critical illness: implications for tight glycemic control," Ann Intensive Care, vol. 2, p. 17, 2012. 